Although antibacterial (e.g. cefepime, cefoperazone/sulbactam and moxifloxacin) and antifungal agents (itraconazole) were administered intravenously according to the microbiological results, the patient had no response and finally died of respiratory failure after a 28-day ICU stay.

The isolation of G100 was unlikely to have been due to contamination, based on the following observations. First, the isolation of Ralstonia spp. from clinical samples is extremely rare at our centre and no other cultures in West China Hospital grew Ralstonia spp. in this year. Secondly, no saline solutions or water were used to collect the sputum sample, which was collected prior to noninvasive ventilation. Thirdly, Gram-negative rods were detected in the sputum by microscopy. As G100 was recovered from a sample collected on admission and this patient had no recent history of hospitalisation, this isolate probably had a community origin. After administration of piperacillin/tazobactam, no $R$. mannitolilytica was isolated from sputum samples collected from this patient afterwards, but the patient's condition did not improve. This suggests that G100 was probably not the causative agent, or at least, not the sole causative agent of the acute exacerbation of COPD in this patient. It is more likely that $R$. mannitolilytica had colonised in the respiratory tract in this case.

R. mannitolilytica has been recovered from the respiratory tract of patients with cystic fibrosis [6]. However, cystic fibrosis is not common in China and this patient did not have this disease. As microbial colonisation of airways could lead to exacerbations of COPD, the isolation of $R$. mannitolilytica in the respiratory tract of the COPD patient could be of clinical importance. As misidentification is common, $R$. mannitolilytica might be an overlooked member of the bacterial flora in the respiratory tract of COPD patients. Ralstonia spp. (identified as R. pickettii, but without describing the method for identification) have previously been found to cause COPD exacerbation [9]. Nonetheless, our report describes a rare case of $R$. mannitolilytica associated with a COPD patient and, to our knowledge, this case is the first identification of $R$. mannitolilytica from clinical samples in mainland China.

\section{Z.Y. Zong* and C.H. Peng",}

*Center of Infectious Diseases, "Dept of Respiratory Medicine, West China Hospital, Sichuan University, Chengdu, and “Dept of Emergency Medicine, People's Hospital of Guizhou Province, Guiyang, China.

Correspondence: Z.Y. Zong, Center of Infectious Diseases, West China Hospital (Huaxi), Guoxuexiang 37, Chengdu 610041, China. E-mail: zongzhiy@scu.edu.cn

Statement of Interest: None declared.

\section{REFERENCES}

1 De Baere T, Steyaert S, Wauters G, et al. Classification of Ralstonia pickettii biovar 3/'thomasii' strains (Pickett 1994) and of new isolates related to nosocomial recurrent meningitis as Ralstonia mannitolytica sp. nov. Int J Syst Evol Microbiol 2001; 51: 547-558.

2 Baird RM, Elhag KM, Shaw EJ. Pseudomonas thomasii in a hospital distilled-water supply. J Med Microbiol 1976; 9: 493-495.

3 Pan HJ, Teng LJ, Tzeng MS, et al. Identification and typing of Pseudomonas pickettii during an episode of nosocomial outbreak. Zhonghua Min Guo Wei Sheng Wu Ji Mian Yi Xue Za Zhi 1992; 25: 115-123.

4 Jhung MA, Sunenshine RH, Noble-Wang J, et al. A national outbreak of Ralstonia mannitolilytica associated with use of a contaminated oxygen-delivery device among pediatric patients. Pediatrics 2007; 119: 1061-1068.

5 Lane DJ. 16S/23S rRNA sequencing. In: Stackebrant E, Goodfellow $\mathrm{M}$, eds. Nucleic acid techniques in bacterial systematics. New York, John Wiley \& Sons, 1991; pp. 115-175.

6 Coenye T, Vandamme P, LiPuma JJ. Infection by Ralstonia species in cystic fibrosis patients: identification of $R$. pickettii and $R$. mannitolilytica by polymerase chain reaction. Emerg Infect Dis 2002; 8: 692-696.

7 Maroye P, Doermann HP, Rogues AM, et al. Investigation of an outbreak of Ralstonia pickettii in a paediatric hospital by RAPD. J Hosp Infect 2000; 44: 267-272.

8 Daxboeck F, Stadler M, Assadian O, et al. Characterization of clinically isolated Ralstonia mannitolilytica strains using random amplification of polymorphic DNA (RAPD) typing and antimicrobial sensitivity, and comparison of the classification efficacy of phenotypic and genotypic assays. J Med Microbiol 2005; 54: 55-61.

9 Sancho-Chust JN, Andreu AL, Chiner E. Ralstonia picketti in chronic obstructive pulmonary disease exacerbation. Arch Bronconeumol 2010; 46: 47-48.

\title{
Can transmissible strains of Pseudomonas aeruginosa be successfully eradicated?
}

\section{To the Editors:}

Recent microbiological surveillance using molecular typing (genotyping) has provided compelling evidence for Pseudomonas aeruginosa cross-infection at many European, Australian and Canadian cystic fibrosis (CF) centres [1-4]. The transmissible strains responsible for this cross-infection pose an increased risk for acquisition of infection for patients currently free of $P$. aeruginosa. As transmissible strains are often resistant to multiple antibiotics, they may also be more difficult to eradicate. At present, there is a paucity of evidence regarding this. We therefore report the efficacy of eradication therapy in a cohort of six patients who acquired a transmissible strain as their first isolate of $P$. aeruginosa. 
$P$. aeruginosa is the most prevalent pulmonary pathogen for individuals with CF. Chronic infection is associated with increased treatment requirements, an accelerated decline in lung function and reduced survival [5]. Aggressive antibiotic treatment can potentially eradicate early $P$. aeruginosa infection and prevent or delay chronic infection. The optimal eradication regimen remains unclear, as published studies all have success rates of $\sim 80 \%$ [6]. The UK Cystic Fibrosis Trust consensus guidelines suggest the use of oral ciprofloxacin and nebulised colistin when P. aeruginosa is first isolated [7]. Paediatric CF centres that have introduced measures to prevent crossinfection and adopted a policy of proactive screening and aggressive treatment of early $P$. aeruginosa infection have reported significant reductions in age-dependent prevalence of $P$. aeruginosa infection [8]. Successful eradication has also been shown to be associated with a reduced deterioration in lung function [6].

A policy of microbiological surveillance for P. aeruginosa crossinfection was introduced at our centre (Manchester Adult Cystic Fibrosis Centre, Wythenshawe Hospital, Manchester, UK) in 2000, including genotyping of all new P. aeruginosa isolates. This demonstrated evidence of cross-infection [1] and new infection control measures were therefore introduced. Patients free from $P$. aeruginosa infection attended a separate outpatient clinic and, when in-patients, used the two single rooms with en suite facilities; they were also advised not to mix with other patients. Infection control measures were further extended in 2004, as continued microbiological surveillance demonstrating ongoing cross-infection [9]. Full isolation measures were introduced for in-patients, and outpatient clinics were further segregated.

The success of eradication therapy for all cases of new acquisition of P. aeruginosa between January 2000 and January 2011 was reviewed. Six patients were identified who had acquired a transmissible strain as their first isolate of $P$. aeruginosa: four cases were identified as the Manchester strain [1] and two as the Liverpool Epidemic strain [2]. The median age at acquisition was 30.5 yrs. The median (range) forced expiratory volume in $1 \mathrm{~s}$ at acquisition was $2.8(0.55-2.95) \mathrm{L}$ and the median forced vital capacity was $3.5(0.9-3.95) \mathrm{L}$. All isolates had a nonmucoid phenotype and exhibited in vitro resistance to one or more antipseudomonal antibiotic (table 1). Only one out of the six patients had their transmissible strain of $P$. aeruginosa successfully eradicated.

A 32-yr-old female acquired $P$. aeruginosa for the first time during a prolonged in-patient admission in June 2000. She was 22 weeks pregnant and admitted with an infective exacerbation complicated by a persistent pneumothorax. 2 weeks into the admission, P. aeruginosa was isolated from her sputum. Genotyping confirmed the nonmucoid, multiresistant isolate to be a transmissible strain (Manchester strain). She remained an in-patient for 6 months and received intravenous antibiotics throughout. After the P. aeruginosa isolation, she received 4 days of nebulised gentamicin and was then commenced on nebulised colistin, which was continued long-term. Despite this, the infection became chronic.

A 29-yr-old male had a first isolation of P. aeruginosa in August 2000, following an in-patient admission for an infective exacerbation. The isolate was nonmucoid and multiresistant; genotyping confirmed it was a transmissible strain (Manchester strain). The patient was commenced on oral ciprofloxacin and nebulised colistin for 1 month. The $P$. aeruginosa persisted, and he was therefore readmitted for a 2-week course of i.v. ceftazidime and tobramycin with nebulised colistin. This failed to eradicate the organism and he became chronically infected.

A 20-yr-old female was admitted in December 2000 with an infective exacerbation; $P$. aeruginosa was isolated for the first time from her sputum. She had been an in-patient on the CF ward 5 months earlier for peripartum i.v. antibiotics prior to a planned delivery. Genotyping confirmed the multiresistant isolate as a transmissible stain of $P$. aeruginosa (Manchester strain). She was admitted for 5 days of $i . v$. ceftazidime and gentamicin. Nebulised colistin was commenced and continued long-term. Despite this, she continued to isolate $P$. aeruginosa. Oral ciprofloxacin was not used as she was known to be intolerant.

A 35-yr-old female became infected with $P$. aeruginosa in June 2003 following an in-patient admission. The nonmucoid, multiresistant isolate was confirmed by genotyping as being a transmissible stain (Liverpool Epidemic strain). She was readmitted for 1 week of i.v. ceftazidime and tobramycin, and then treated with oral ciprofloxacin and nebulised colistin for 1 month. Persistence of $P$. aeruginosa was noted and nebulised tobramycin was used for a further month in a further attempt at eradication. However, the patient developed chronic $P$. aeruginosa infection.

A 43-yr-old female developed $P$. aeruginosa infection in April 2004 during an in-patient admission for an infective exacerbation. She was on long-term nebulised gentamicin for chronic Staphylococcus aureus infection and nebulised colistin, which she chose to continue despite not having isolated $P$. aeruginosa in the $13 \mathrm{yrs}$ in which she had attended our unit. The $P$. aeruginosa isolate was nonmucoid and multiresistant; genotyping confirmed it as a transmissible strain (Liverpool Epidemic strain). She received 18 days of i.v. ceftazidime, meropenem and tobramycin. Nebulised gentamicin and colisitin were continued, and oral ciprofloxacin commenced. The ciprofloxacin had to be discontinued, as it was not tolerated. Despite this treatment, the patient became chronically infected.

A 21-yr-old female developed P. aeruginosa infection in December 2009. Genotyping identified the nonmucoid, multiresistant isolate as a transmissible strain (Manchester stain). Previously, she had exclusively attended the non-Pseudomonas outpatient clinic. There had been no recent hospital admissions and no known contact with other CF patients. She was admitted for 2 weeks of i.v. ceftazidime and tobramycin. As she was intolerant to oral ciprofloxacin, she received 4 weeks of nebulised tobramycin and then changed to nebulised colistin, which was continued long-term. In the subsequent 13 months, 20 sputum samples have been cultured and none have isolated $P$. aeruginosa.

Despite aggressive treatment, only one (17\%) out of six patients who had acquired a transmissible strain as their first isolate of $P$. aeruginosa had it successfully eradicated. This is in contrast to the widely accepted success rate for $P$. aeruginosa eradication of $80 \%$ [6]. This implies that multiresistant transmissible strains of $P$. aeruginosa present an increased risk both of acquisition of infection and subsequent progression to chronic infection. 
TABLE 1 Patient demographic data, antibiogram and eradication regimens

\begin{tabular}{|c|c|c|c|c|c|c|}
\hline Date of isolate & June 2000 & August 2000 & December 2000 & June 2003 & April 2004 & December 2009 \\
\hline Sex & Female & Male & Female & Female & Female & Female \\
\hline BMI $\mathbf{k g} \cdot \mathrm{m}^{-2}$ & 20 & 23 & 32 & 20 & & 21 \\
\hline FEV 1 L & 1.3 & 2.8 & 2.9 & 2.95 & 0.55 & 2.85 \\
\hline \multicolumn{7}{|l|}{ First $P$. aeruginosa isolate } \\
\hline Strain & Manchester & Manchester & Manchester & Liverpool Epidemic & Liverpool Epidemic & Manchester \\
\hline Phenotype & Nonmucoid & Nonmucoid & Nonmucoid & Nonmucoid & Nonmucoid & Nonmucoid \\
\hline \multicolumn{7}{|l|}{ Antibiogram of first isolate } \\
\hline Aztreonam & R & $\mathrm{R}$ & $\mathrm{R}$ & $\mathrm{R}$ & $\mathrm{R}$ & $\mathrm{R}$ \\
\hline Meropenem & $\mathrm{R}$ & $\mathrm{R}$ & R & $R$ & $\mathrm{R}$ & R \\
\hline Tazocin & $\mathrm{R}$ & $\mathrm{R}$ & S & S & S & $R$ \\
\hline Tobramycin & S & S & S & । & S & S \\
\hline \multicolumn{7}{|l|}{ Eradication regimen } \\
\hline $\begin{array}{l}\text { Intravenous antibiotics } \\
\text { (days) }\end{array}$ & $\begin{array}{l}\text { Combination of ceftazidime/ } \\
\text { tobramycin/colistin/tazocin/ } \\
\text { meropenem/cotrimoxazole/ } \\
\text { imipenim (189) }\end{array}$ & $\begin{array}{l}\text { Ceftazidime (14) } \\
\text { Tobramycin (14) }\end{array}$ & $\begin{array}{l}\text { Ceftazidime (5) } \\
\text { Gentamicin (5) }\end{array}$ & $\begin{array}{l}\text { Ceftazidime (7) } \\
\text { Tobramycin (7) }\end{array}$ & $\begin{array}{l}\text { Ceftazidime (18) } \\
\text { Tobramycin (18) } \\
\text { Meropenem (18) }\end{array}$ & $\begin{array}{l}\text { Ceftazidime (14) } \\
\text { Tobramycin (14) }\end{array}$ \\
\hline Oral antibiotics (days) & & Ciprofloxacin (28) & & Ciprofloxacin (28) & Ciprofloxacin ${ }^{\#}$ & \\
\hline Nebulised antibiotics (days) & $\begin{array}{l}\text { Gentamicin (4) } \\
\text { Colistin (cont.) }\end{array}$ & Colistin (cont.) & Colistin (cont.) & $\begin{array}{l}\text { Colistin (28) then } \\
\text { tobramycin (28) }\end{array}$ & $\begin{array}{c}\text { Colistin (cont.) } \\
\text { Gentamicin (cont.) }\end{array}$ & $\begin{array}{l}\text { Tobramycin (28) } \\
\text { then colistin (cont.) }\end{array}$ \\
\hline
\end{tabular}

BMI: body mass index; FEV1: forced expiratory volume in $1 \mathrm{~s}$; FVC: forced vital capacity; P. aeruginosa: Pseudomonas aeruginosa; S. aureus: Staphylococcus aureus; R: resistant; I: inhibitory; S: sensitive; cont.: continuous; A. xylosoxidans: Alcaligenes xylosoxidans. ${ }^{*}$ : discontinued due to intolerance.

Five of the patients in this series acquired transmissible $P$. aeruginosa following in-patient admission, suggesting crossinfection between patients on the CF ward. All of these occurred prior to the full isolation measures being introduced at our unit. Since these infection prevention measures were introduced, we have not had any patients acquiring a transmissible strain as their first isolate of $P$. aeruginosa following in-patient admission. The source of the transmissible strain that infected patient 6 is unclear, as she had no recent hospital admissions and no known contact with other CF patients. Two of the females acquired infection with a transmissible strain during pregnancy, raising the question of whether this is a time of increased susceptibility to $P$. aeruginosa infection.

The exact reason transmissible strains of $P$. aeruginosa are more difficult to eradicate is unclear. It is likely that antibiotic resistance has some influence on this, as the multiresistance exhibited by transmissible strains of $P$. aeruginosa is in contrast to that of new infection with sporadic strains, which tend to be sensitive to usual antipseudomonal antibiotics [10]. This highlights the need to genotype first isolates, particularly if they exhibit antibiotic resistance. However, antibiotic resistance may not be the only explanation, as the transmissible strain that was successful eradicated in patient 6 was also multiresistant. It is known that transmissible strains of $P$. aeruginosa have a number of unusual phenotypic features [1], and it is possible that they have developed survival mechanisms to avoid eradication and quickly establish chronic infection.

In summary, transmissible strains present an increased risk to patients who are free of $P$. aeruginosa infection of developing chronic $P$. aeruginosa infection both through an increased risk of initial acquisition associated with cross-infection and also failure of established eradication treatment to clear the early infection. Prevention can only be achieved by the implementation of infection control measures and the success of these measures should be judged by continued microbiological surveillance.

\section{F.J. Gilchrist*, M. France*, R. Bright-Thomas*, C.J. Doherty J.R. Govan", A.K. Webb* ${ }^{*}$ and A.M. Jones ${ }^{*}$,}

*Manchester Adult Cystic Fibrosis Centre, Wythenshawe Hospital, "Respiratory Research Group, Faculty of Medical and Human Sciences, The University of Manchester, Manchester Academic Health Science Centre, Manchester, and ${ }^{\#}$ Centre for Infectious Diseases, New Royal Infirmary, University of Edinburgh, Edinburgh, UK. 
Correspondence: A.M. Jones, Manchester Adult Cystic Fibrosis Centre, Wythenshawe Hospital, Southmoor Road, Manchester M23 9LT, UK. E-mail: andrew.jones@uhsm.nhs.uk

Statement of Interest: None declared.

\section{REFERENCES}

1 Jones AM, Govan JR, Doherty CJ, et al. Spread of a multiresistant strain of Pseudomonas aeruginosa in an adult cystic fibrosis clinic. Lancet 2001; 358: 557-558.

2 Cheng K, Smyth RL, Govan JR, et al. Spread of $\beta$-lactam-resistant Pseudomonas aeruginosa in a cystic fibrosis clinic. Lancet 1996; 348: 639-642.

3 Armstrong DS, Nixon GM, Carzino R, et al. Detection of a widespread clone of Pseudomonas aeruginosa in a pediatric cystic fibrosis clinic. Am J Respir Crit Care Med 2002; 166: 983-987.

4 Aaron SD, Vandemheen KL, Ramotar K, et al. Infection with transmissible strains of Pseudomonas aeruginosa and clinical outcomes in adults with cystic fibrosis. JAMA 2010; 304: 2145-2153.
5 Emerson J, Rosenfeld M, McNamara S, et al. Pseudomonas aeruginosa and other predictors of mortality and morbidity in young children with cystic fibrosis. Pediatr Pulmonol 2002; 34: 91-100.

6 Jones AM. Eradication therapy for early Pseudomonas aeruginosa infection in CF: many questions still unanswered. Eur Respir J 2005; 26: 373-375.

7 UK Cystic Fibrosis Trust Antibiotic Working Group. Antibiotic Treatment for Cystic Fibrosis. 3rd Edn. Bromley, Cystic Fibrosis Trust, 2009.

8 Lee TWR, Brownlee KG, Denton M, et al. Reduction in prevalence of chronic Pseudomonas aeruginosa infection at a regional paediatric cystic fibrosis center. Pediatr Pulmonol 2004; 37: 104-110.

9 Jones AM, Dodd ME, Govan JRW, et al. Prospective surveillance for Pseudomonas aeruginosa cross-infection at a cystic fibrosis center. Am J Respir Crit Care Med 2005; 171: 257-260.

10 Macdonald D, Cuthbertson L, Doherty C, et al. Early Pseudomonas aeruginosa infection in individuals with cystic fibrosis: is susceptibility testing justified? J Antimicrob Chemother 2010; 65: 2373-2375.

\section{Diagnosis of respiratory viral infections in cystic fibrosis by PCR using sputum samples}

\section{To the Editors:}

Recent epidemics of virulent respiratory viruses, such as H1N1 influenza and severe acute respiratory syndrome coronavirus (SARS-CoV), have highlighted the clinical importance of methods for the rapid and accurate detection of respiratory virus infection $[1,2]$. A diagnosis of a respiratory viral infection may have implications for infection control measures and treatment of patients [3]. Molecular-based techniques offer a more rapid and sensitive method for diagnosis of respiratory viral infection than viral culture or serology [4]. PCR-based methods can be applied to nasal and/or throat swab samples for the rapid detection of common respiratory viruses [5]. As the majority of adults with cystic fibrosis (CF) produce sputum during infective exacerbations, we investigated whether sputum is a suitable medium for the diagnosis of respiratory viral infections in patients with $\mathrm{CF}$ using a PCR method, by comparing results of PCR tests from paired sputum and nasal swab samples collected from patients with symptoms of a viral respiratory illness.

We prospectively collected paired nasal swab and sputum samples from adult patients with CF who presented with symptoms of a respiratory viral illness during the period December 2008 to June 2009. Flocked nasal swab samples were transported to the laboratory in viral transport medium (Microtest ${ }^{\mathrm{TM}} \mathrm{M}^{\mathrm{R}} \mathrm{RT}_{\text {}}$; Remel, Lenexa, KS, USA).

The samples were investigated using an in-house PCR method to detect common respiratory viruses (rhinovirus, influenza A, influenza B, parainfluenza types 1-3, adenovirus, respiratory syncytial virus (RSV) and metapneumovirus).
Permission for the study was granted by the local medical ethics committee. All patients gave verbal informed consent for participation in the study.

Group results were compared by a McNemar test using the SPSS statistical package, version 15 (SPSS Inc., Chicago, IL, USA).

Paired samples were analysed from 53 patients. 25 (47.2\%) patients had positive results with 26 viruses detected: 13 rhinovirus, three influenza A, three influenza B, three parainfluenza type 3, two adenovirus, one RSV and one metapneumovirus. Of the 25 positive results, all (100\%) sputum samples were positive, whilst $17(68 \%)$ nasal swab samples were positive $(p=0.008)$. One patient had both positive nasal and sputum samples for rhinovirus, but the sputum sample was also positive for adenovirus.

The importance of rapid, specific and sensitive tests for respiratory viral infections has been highlighted by recent epidemics of virulent respiratory viruses, such as $\mathrm{H} 1 \mathrm{~N} 1$ pandemic influenza [1] and SARS-CoV [2]. This study has shown that sputum, which is a readily available sample, is a suitable specimen for analysis by PCR for the rapid diagnosis of common respiratory viral infections. A previous study also reported success in detection of virus infection using a PCR technique with sputum samples for patients with $\mathrm{CF}$, but did not compare yield with traditional nasal samples [6]. In our clinical practice, the sensitivity of PCR is greater using sputum than nasal swab samples.

Tissue culture and serology were previously used as the standard tools for screening for viral infections, but have 